\title{
The Role of Strange Quasiquarks in Transport Properties of the QGP
}

\author{
Valeriya Mykhaylova ${ }^{1, *}$ \\ ${ }^{1}$ Institute of Theoretical Physics, University of Wrocław, PL-50204 Wrocław, Poland
}

\begin{abstract}
We study the role of dynamical quarks in the transport properties of the deconfined hot medium utilizing the quasiparticle kinetic approach. The system is then composed of the quasiparticle excitations, whose interactions are encoded in their dynamical masses with temperature dependence specified by the effective coupling extracted from lattice QCD thermodynamics. Evaluating the temperature and flavor profiles of the shear and bulk viscosities, as well as the electrical conductivity we examine how different particle species, in particular strange quarks, modify the transport parameters of the deconfined matter.
\end{abstract}

\section{Introduction}

One of the major aims of the experimental and theoretical studies of the quark-gluon plasma (QGP) is to reveal its dynamical and transport properties, quantified by various transport parameters.

The shear viscosity $\eta$ measures the resistance of the fluid against momentum modifications during its longitudinal motion [1], while the bulk viscosity $\zeta$ indicates the energy dissipation caused by the expansion of the QGP [2]. Their dimensionless ratios to the entropy density, i.e. $\eta / s, \zeta / s$ are the major input in the hydrodynamic equations [3]. The other important parameter is the electrical conductivity $\sigma$, since the strong electric and magnetic fields are expected to emerge in the non-central heavy-ion collisions. A precise determination of transport coefficients as functions of temperature and chemical potential is one of the main steps towards understanding the non-trivial evolution of strongly interacting matter.

For a comprehensive study of the bulk properties of the deconfined matter conducted within the quasiparticle model (QPM), we refer the reader to $[1,2,4]$. In this work, we summarize our numerical results on transport coefficients at finite temperature and vanishing chemical potential. We assess the influence of dynamical quasiparticles on the evolution of different coefficients, focusing particularly on the role of the strange quark flavor. In Sec. 2, we briefly discuss the main assumptions of the QPM. In Sec. 3, we present the results for transport coefficients derived in kinetic theory under the relaxation time approximation. A summary and conclusions are given in Sec. 4.

\section{Quasiparticle Model}

We examine transport properties of the QGP within the well-grounded quasiparticle model $[1,5]$. In this aproach the QGP is described as a viscous fluid composed of quasiparticles, whose dynamical masses $m_{i}(T)$ arise as they propagate through the system,

\footnotetext{
*e-mail: valeriya.mykhaylova@uwr.edu.pl
} 
$m_{i}^{2}(T)=\left(m_{i}^{0}\right)^{2}+\Pi_{i}(T)$. The effective mass depends on the bare particle mass $m_{i}^{0}$ and the gauge-independent self-energy $\Pi_{i}$ defined as [5]

$$
\Pi_{l, s}(T)=2\left(m_{l, s}^{0} \sqrt{\frac{G(T)^{2}}{6} T^{2}}+\frac{G(T)^{2}}{6} T^{2}\right), \quad \Pi_{g}(T)=\left(3+\frac{N_{f}}{2}\right) \frac{G(T)^{2}}{6} T^{2} .
$$

The effective running coupling $G(T)$ is deduced from the equation of state calculated in lattice gauge theory for the QGP with $2+1$ quark flavors and the gluon plasma in pure Yang-Mills theory. This way, the $G(T)$ incorporates the non-perturbative dynamics near the QCD phase transition and reproduces the perturbative behavior in the very high-temperature regime $[1,2]$.

\section{Transport parameters in kinetic theory}

Assuming that a system slightly deviates from thermal equilibrium, we employ the expressions for the transport parameters determined from kinetic theory under the relaxation time approximation. The derivation and detailed analysis are presented in $[1,5,6]$ for the shear viscosity $\eta$, in $[2,5]$ for the bulk viscosity $\zeta$, and in $[2,4,7]$ for the electrical conductivity $\sigma$.
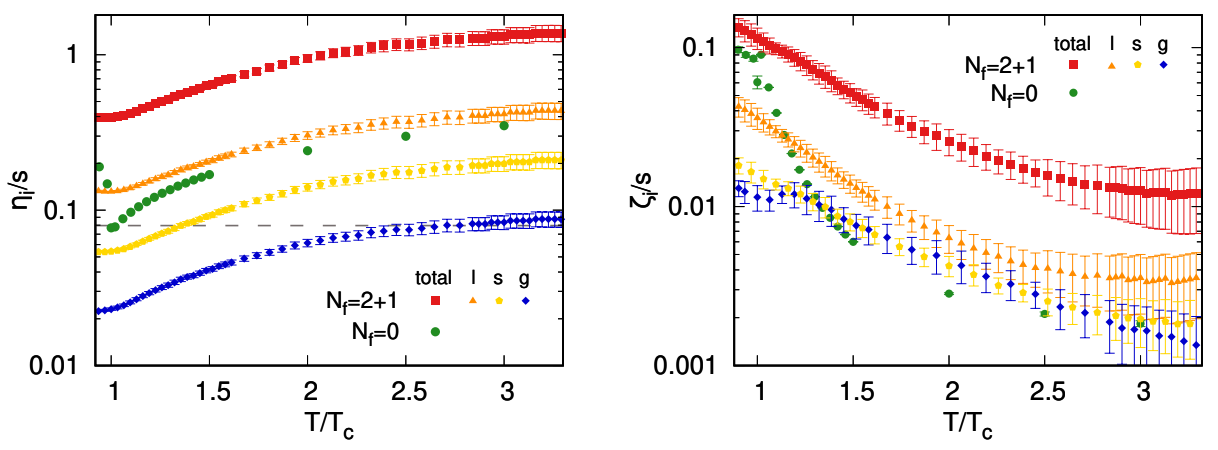

Figure 1. Shear (left) and bulk (right) viscosities to the total entropy density ratios as functions of $T / T_{c}$. The individual contributions from gluons (diamonds), light (triangles) and strange (pentagons) quarks are shown along with the total ratios for the QGP with $N_{f}=2+1$ (squares) and $N_{f}=0$ (circles). In the left panel, the Kovtun-Son-Starinets (KSS) bound of $1 / 4 \pi$ is indicated by the dashed line.

In Fig. 1 we present the total specific shear and bulk viscosities of the hot QCD medium, along with the individual contributions of each quasiparticle sector in case of $N_{f}=2+1$. The hierarchy in $\eta_{i} / s$ follows the ordering in the effective masses [1], while in $\zeta_{i} / s$ this behavior is disturbed. Although strange quarks and gluons are described by different statistical distributions, there appears a quantitative resemblance between $\zeta_{s} / s$ and $\zeta_{g} / s$ ratios. We find that the similarity arises due to a convolution of the factors entering the expression for the bulk viscosity [2]. Overall, the light quarks bring the main impact to the total shear and bulk viscosities, while the heaviest quasiparticles are the most effective ones in equilibrating momentum degradations within the QGP.

Compared to the pure Yang-Mills theory, the presence of dynamical quarks in QCD significantly increases $\eta / s$ and $\zeta / s$ ratios. Further, one clearly observes a smooth behavior of the specific viscosities in the vicinity of the QCD crossover, and a strong non-monotonicities around $T_{c}$ in pure Yang-Mills theory due to the first-order phase transition [1]. 
Fig. 2 (left) illustrates the bulk to shear viscosity ratios $\zeta_{i} / \eta_{i}$ obtained by dividing each curve presented on the left side of Fig. 1 by the corresponding curve on the right. We have utilized the total $\zeta / \eta$ ratio in QCD and pure Yang-Mills theory to study the impact of quarks onto the deviation of the deconfined matter from conformal invariance [2]. Here, we observe an interesting overlap of the $\zeta_{s} / \eta_{s}$ component coming from strange quarks, with the total $\zeta / \eta$ ratio. Thus, the strange quark sector quantitatively resembles the bulk to shear viscosity ratio for the QGP. The contribution from the light quark sector slightly underestimates the total $\zeta / \eta$ ratio, while the $\zeta_{g} / \eta_{g}$ is much larger due to the suppressed gluonic term in the shear viscosity, see left panel of Fig. 1.
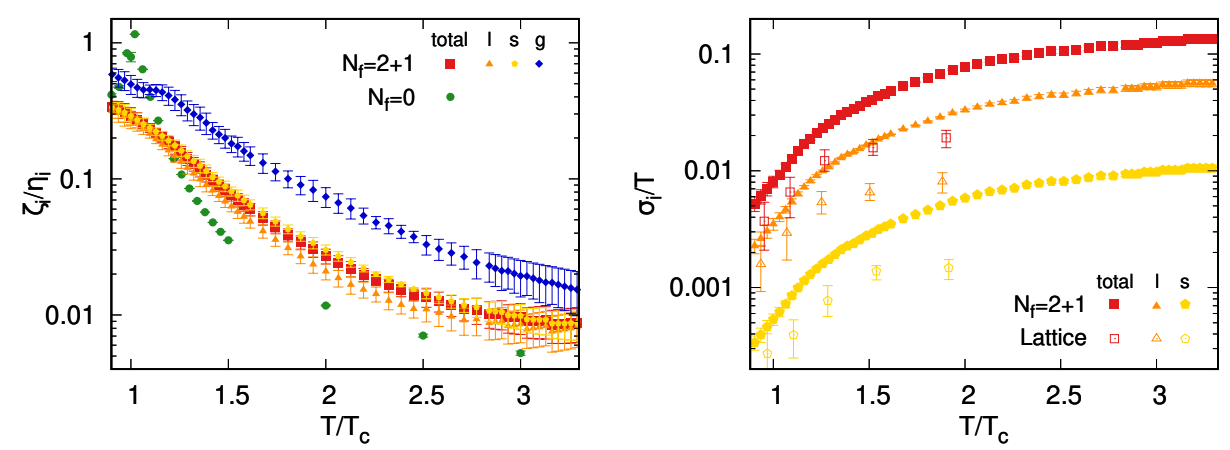

Figure 2. Left: Bulk to shear viscosity ratio for the QGP with $N_{f}=2+1$ (squares), shown along with the individual contributions from light quarks (triangles), strange quarks (pentagons) and gluons (diamonds). Alongside, the ratio for pure gluon plasma (circles) is presented. Right: The scaled electrical coductivity $\sigma_{i} / T$ for the QGP (full squares) along with the light (sum of up and down, full triangles) and strange (full pentagons) quark components. The corresponding lattice data is deduced from [8] for the total electrical conductivity (open squares) and for light and strange quark contributions (open triangles and open pentagons, respectively).

In the right panel of Fig. 2 we present the total scaled electrical conductivity $\sigma / T$ along with the contributions from light and strange quasiparticle sectors. The light-quark contribution is larger than that of the strange quarks since it contains electric charges of up and down quarks and since the strange quark component is suppressed by the larger effective mass. The corresponding lattice data [8] near $T_{c}$ is rather compatible with the QPM result, whereas the discrepancy between them emerges at $T \simeq 1.5 T_{c}$ and increases gently with temperature. This can be attributed to the fact that the lattice setup includes the pion mass $M_{\pi}=384(4) \mathrm{MeV}$, heavier than the physical one used in our model-building.

For a detailed comparison of the total $\sigma / T$ ratio with the results computed in other approaches, we refer a reader to $[2,4]$.

\section{Conclusions}

We have examined the influence of different dynamical particles, especially the strange quarks, on transport coefficients of the deconfined matter. The transport parameters were computed within kinetic theory under the relaxation time approximation for a medium approached by the quasiparticle model (QPM). The dynamical masses of quasiparticles are characterized by the effective temperature-dependent running coupling deduced from the entropy density obtained by lattice simulations. 
Assuming that the transport parameters are characterized by the common relaxation time for each quasiparticle species, we computed the temperature and flavor dependence of the specific shear $\eta / s$ and bulk $\zeta / s$ viscosities, and of the scaled electrical conductivity $\sigma / T$.

We juxtaposed the total results for the QGP with $N_{f}=2+1$ with the transport parameters of the gluon plasma in pure Yang-Mills theory. Above the (pseudo)critical temperature, all transport parameters of the pure gluon plasma are much smaller than that for the QGP with dynamical quarks. Thus, the presence of dynamical quarks significantly changes the transport properties of the deconfined matter.

For the QGP with quark quasiparticles, we found that the major contribution to the transport coefficients comes from the light-quark sector, while the components from strange quarks and gluons are suppressed by their larger effective masses. While for the specific shear viscosity $\eta / s$ we observed a clear ordering in the contributions from different quasiparticles, for the bulk viscosity $\zeta / s$ we found that the strange quarks and gluons contribute to the total ratio in almost the same amount due to the convolution of their observables, such as effective masses and relaxation times. The bulk to shear viscosity ratio and its components reveal that in the QPM, the $\zeta_{s} / \eta_{s}$ ratio coming from the strange quark sector quantitatively resembles the total $\zeta / \eta$ ratio of the QGP with $2+1$ quark flavor.

Finally, the individual contributions from light and strange quarks to the electrical conductivity $\sigma / T$ were confronted with the total ratio and the corresponding lattice data. We found our results to be qualitatively consistent with the recent lattice QCD outsomes [8].

\section{Acknowledgments}

The author thanks Chihiro Sasaki for a scientific advisory and fruitful collaboration. This work was partly supported by the Polish National Science Center (NCN) under the Opus grant no. 2018/31/B/ST2/01663.

\section{References}

[1] V. Mykhaylova, M. Bluhm, K. Redlich and C. Sasaki, Phys. Rev. D 100, no.3, 034002 (2019) doi:10.1103/PhysRevD.100.034002.

[2] V. Mykhaylova and C. Sasaki, Phys. Rev. D 103, no.1, 014007 (2021) doi:10.1103/PhysRevD.103.014007.

[3] P. Huovinen, P. Kolb, U. W. Heinz, P. Ruuskanen and S. Voloshin, Phys. Lett. B 503, 58-64 (2001) doi:10.1016/S0370-2693(01)00219-2.

[4] V. Mykhaylova, Eur. Phys. J. ST 229, no.22-23, 3487-3496 (2020) doi:10.1140/epjst/e2020-000116-9.

[5] M. Bluhm, B. Kämpfer and K. Redlich, Nucl. Phys. A 830, 737C-740C (2009) doi:10.1016/j.nuclphysa.2009.10.065.

[6] C. Sasaki and K. Redlich, Phys. Rev. C 79, 055207 (2009) doi:10.1103/PhysRevC.79.055207.

[7] L. Thakur, P. K. Srivastava, G. P. Kadam, M. George and H. Mishra, Phys. Rev. D 95, no. 9, 096009 (2017) doi:10.1103/PhysRevD.95.096009.

[8] G. Aarts, C. Allton, A. Amato, P. Giudice, S. Hands and J. I. Skullerud, JHEP 02, 186 (2015) doi:10.1007/JHEP02(2015)186. 